\title{
Ryanodine Receptors Coupling Causes a Calcium Leak in Cardiac Cell
}

\author{
Alexander Ryvkin ${ }^{1,2}$, Nikita Markov $^{2}$ \\ ${ }^{1}$ Institute of Immunology and Physiology UrB RAS \\ ${ }^{2}$ Ural Federal University, Ekaterinburg, Russia
}

\begin{abstract}
Here we introduce results of a mathematical modeling of calcium sparks in cardiac cells. We developed a model of the calcium release unit which includes a single sarcoplasmic reticulum (SR) lumen, a regular $9 x 9$ cluster of RyRs and a dyadic space. 2D diffusion problem of $\mathrm{Ca} 2+$ ions across the dyadic space was solved thereby we reproduced Calcium-Induced-Calcium-Release (CICR) effect and domino-like RyRs activation in the cluster.

We take into account allosteric and $\mathrm{Ca}^{2+}$-induced coupling between RyRs. We show, that coupling between RyRs leads to the stability of $\mathrm{Ca}^{2+}$ sparks in amplitude and frequency. However, a sudden stop of spontaneous $\mathrm{Ca}^{2+}$ releases can be a result of strong allosteric coupling between RyRs.
\end{abstract}

\section{Introduction}

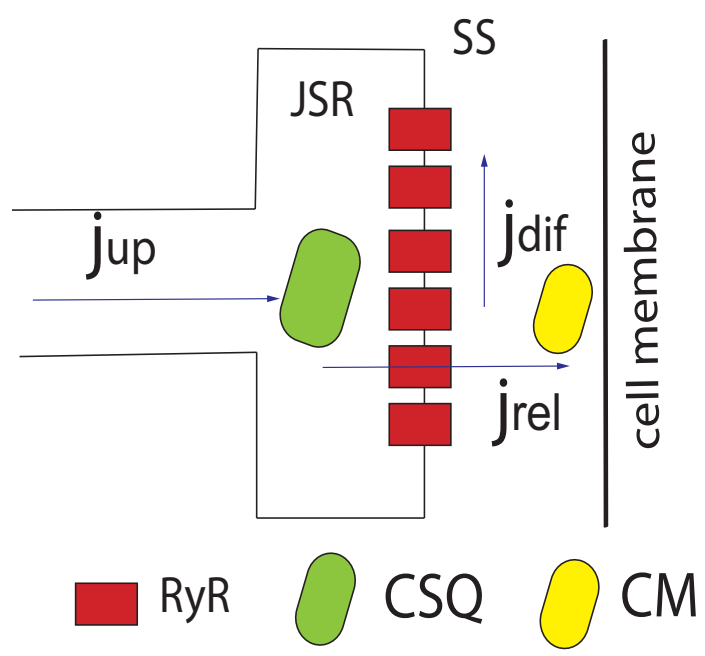

Figure 1. Schematic illustration of $\mathrm{Ca}^{2+}$ currents in the $\mathrm{Ca}^{2+}$-release unit.

Local $\mathrm{Ca}^{2+}$ releases (so-called calcium sparks) from the sarcoplasmic reticulum (SR) are in the basis of a global $\mathrm{Ca}^{2+}$ release process which increases intracellular cal- cium level by an order of magnitude [1].

In the sinoatrial node cells (SANCs) spontaneousCa ${ }^{2+}$ releases play a major role in the action potential generation, thus, the study of the functioning of different parts of the $\mathrm{Ca}^{2+}$ release system is the important problem of the mathematical modeling. Traditionally calcium release system is described within the framework of the "Common Pool" model [2], which summarizes a complex $\mathrm{Ca}^{2+} \mathrm{re}-$ lease system to a single $\mathrm{Ca}^{2+}$ release unit (RU) (Fig. 1). The RU consists of a single lumen (junctial sarcoplasmic reticulum, jSR) and a subspace (SS). There is a compact cluster of $\mathrm{Ca}^{2+}$ releasing ryanodine receptors (RyRs). The regularity of the channel lattice is questionable $[3,4]$; however, the researchers cope with the conclusion that there is both an allosteric and conformational interaction between closely enough located channels [3,5].

$\mathrm{Ca}^{2+}$-mediated, allosteric or conformational coupling between RyRs cause a cooperative effect of RyRs opening and closure and further spark formation. By means of computer modeling we tried to find out which mechanism of interaction can lead to $\mathrm{Ca}^{2+}$ leak from the SR.

$\mathrm{Ca}^{2+}$ ions released via RyRs can activate nearest neighbors in "domino-like" style ( $\mathrm{Ca}^{2+}$-mediated coupling), so this process also amplifies the $\mathrm{Ca}^{2+}$-release. Thus, $\mathrm{Ca}^{2+}$ diffusion in the subspace attracts considerable interest due to the complex RyRs activation process as well as the spark initiation and spread.

In our computer simulations we adopted the formalism of the Maltsev-Lakatta model [6] of the rabbit SANC functioning, taking into account $\mathrm{Ca}^{2+}$-mediated and allosteric coupling between RyRs in the cluster. Describing calcium dynamics we need to take into account $\mathrm{Ca}^{2+}$-binding proteins (buffers): calmodulin and calsequestrin which cause a delay of $\mathrm{Ca}^{2+}$ dynamics in subspace and in jSR (Fig.1).

\section{Methods}

\subsection{Model of calcium dynamics in the car- diac cell}

In our model we take into account a single RU. $\mathrm{Ca}^{2+}$ dynamics is described by the system of reaction-diffusion 
equations:

$$
\begin{aligned}
& \frac{d C a_{S S}}{d t}=\frac{V_{j S R}}{V_{S S}} j_{r e l}-C M_{t o t} \cdot \frac{d f_{C M}}{d t} \\
& \frac{d C a_{j S R}}{d t}=j_{r e f i l l}-j_{r e l}-C Q_{t o t} \cdot \frac{d f_{C Q}}{d t} \\
& \frac{d f_{C M}}{d t}=k_{f C M} C a_{S S}\left(1-f_{C M}\right)-k_{b C M} f_{C M} \\
& \frac{d f_{C Q}}{d t}=k_{f C Q} C a_{j S R}\left(1-f_{C Q}\right)-k_{b C Q} f_{C Q},
\end{aligned}
$$

where $j_{\text {refill }}$ is the lumen refill flux (constant in the current model), $j_{r e l}$ is a release flux via open RyRs, $V_{S S}$ and $V_{j S R}$ are volumes of the subspace and the lumen respectively, $f_{C Q}$ and $f_{C M}$ are current concentrations of a bound calsequestrin and calmodulin respectively, $C Q_{t o t}$ and $C M_{t o t}$ are total concentrations of calsequestrin and calmodulin respectively.

The release flux depends on the number of open RyRs: $j_{r e l}=N_{o p e n} k_{r e l}\left(C a_{j S R}-C a_{S S}\right)$, where $k_{r e l}$ is the release rate constant via a single RyR, $N_{\text {open }}$ is the number of open RyRs .

\subsection{Subspace $\mathrm{Ca}^{2+}$ diffusion model}

In the current work we solve $2 \mathrm{D} \mathrm{Ca}^{2+}$ diffusion problem across the subspace. In our model SR has a cluster of 9x9 RyRs.

$$
\frac{\partial u}{\partial d t}=d \cdot\left(\frac{\partial^{2} u}{\partial x^{2}}+\frac{\partial^{2} u}{\partial y^{2}}\right),
$$

where $u$ is the local $\left(C a_{S S}\right.$ concentration in each node of the mesh. $d$ is a diffusion constant.

We use an implicit finite-difference five-point stencil numerical scheme utilized for approximation of the diffusion equation. Parallel implementation on $\mathrm{C}++$ with the use of PETSc makes it possible.

Our model describes $\mathrm{Ca}^{2+}$ fluxes between the RU compartments and the $\mathrm{Ca}^{2+}$ diffusion in the subspace.

\subsection{RyRs stochastic dynamics model}

Stochastic behavior of RyRs is described in our work in terms of previously developed Electron-Conformational model (ECM) [7,8]. This theory assumes that the RyR has only two degrees of freedom: slow conformational (refers to RyRs conformatioal opening/closure processes) and fast electronic (corresponds to $\mathrm{Ca}^{2+}$ ions effect on RyRs activational sites). RyRs states are described within the framework of elecrton-conformational potential formalism 1:

$$
E_{ \pm}\left(Q_{m}\right)=\frac{K}{2} Q_{m}^{2}-p Q_{m} \pm \frac{1}{2} a Q_{m}+\frac{1}{2} k \sum_{n=1}^{4} Q_{m} Q_{n}
$$

where $Q$ is a conformational coordinate, $a$ is an electronconformational coupling parameter, $p$ is a parameter of an effective "pressure" of the lumen $\mathrm{Ca}^{2+}, K$ is the RyRs effective "elastic" constant. $k$ is the conformational coupling parameter. Electron-conformational potential has two minima 2, left minimum corresponds to the closed state, right to the open. The probability of the interbranch transition between states depends on the $\mathrm{Ca}^{2+}$ concentration near each RyR:

$$
P_{\text {elect }}=\alpha \cdot C a_{S S},
$$

where $\alpha$ is a coefficient of proportionality.

The ECM introduce a novel approach of the description of the RyRs allosteric coupling with their nearest neighbours. In 3 last term describes this kind of interactions with the coupling parameter $k$. As can be seen from 2 the shape of the potential changes, the minimum corresponding to the closed state of the channel becomes more global (Fig.2).

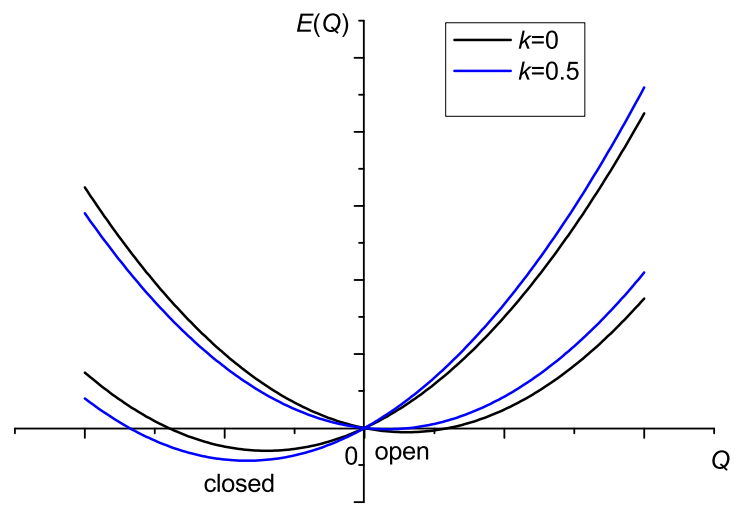

Figure 2. Electron-conformational potential of the RyR. Blue line corresponds to the electron-conformational potential with the allosteric coupling with the nearest closed neighbours.

\section{Results}

A series of computer experiments for the modeling of the $\mathrm{Ca}^{2+}$ release process and RyRs activation was performed. A standard set of the model parameters was taken from the $\mathrm{Ca}^{2+}$-dynamics model in the rabbit pacemaker cell [6] to compare our previous simulation results [9] with the averaged $\mathrm{Ca}^{2+}$ and buffer concentrations in the current work: $k_{b C M}=0.542 \mathrm{~ms}^{-1}, k_{b C Q}=0.445 \mathrm{~ms}^{-1}$; $k_{f C M}=227.7 \mu \mathrm{M}^{-1} \mathrm{~ms}^{-1} ; k_{f C Q}=0.534 \mu \mathrm{M}^{-1} \mathrm{~ms}^{-1}$; $C Q_{\text {tot }}=10 \mu \mathrm{M} ; C M_{\text {tot }}=0.045 \mu \mathrm{M} ; d=10^{-10} \mathrm{~m}^{2} / \mathrm{s}$, $V_{j S R} / V_{S S}=1.6$.

Parameters of the computational method. Number of mesh nodes $m_{x}=m_{y}=240$; a single RyR width $L_{R y R}=37 \mathrm{~nm}$, size of a single mesh node $L_{m e s h}=1 \mathrm{~nm}$, timestep $d t=0.01 \mathrm{~ms}$. $\mathrm{Ca}^{2+}$ concentrations initial values $C a_{j S R}(t=0)=1 \mu \mathrm{M}, C a_{S S}(t=0)=0 \mu \mathrm{M}$, $N_{\text {openrel }}(t=0)=0$. 


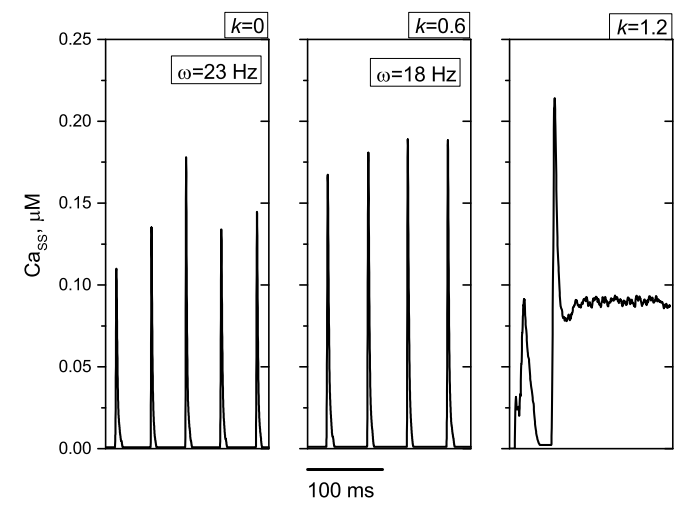

Figure 3. Timeseries of the mean subspace $\mathrm{Ca}^{2+}$ concentration $C a_{S S}$ for different values of the RyRs allosteric coupling $k$.

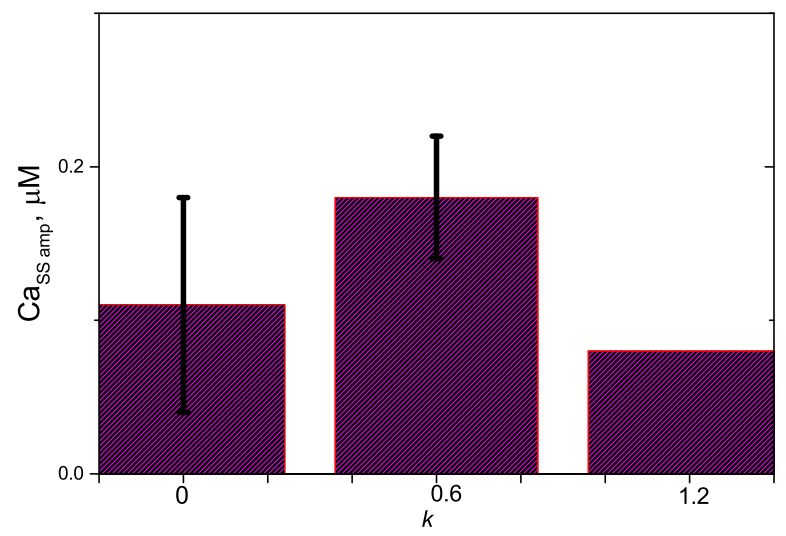

Figure 4. Calcium sparks amplitude $C a_{S \text { Samp }}$ for different values of the RyRs allosteric coupling $k$. Lines show deviations from the mean sparks amplitude.

Electron-conformational model parameters $a=5, K=$ $12, K_{C a}=500 \mu \mathrm{M}, C a_{S_{S \_} \text {crit }}=100 \mu \mathrm{M}, \alpha=$ $0.0012 \mathrm{~ms}^{-1} \mu \mathrm{M}^{-1}$.

Without taking into account $\mathrm{Ca}^{2+}$ diffusion in the subspace, previously, it was shown [9] during computer simulations that the conformational coupling between RyRs in the RU can serve as a stabilizing factor. The strengthening of the conformational cooperativity $(k=1)$ determines the stability of the $\mathrm{Ca}^{2+}$-clock oscillatory dynamics, as well as fluctuations of the $C a_{S S}$ frequency and amplitude. The study of violations of the functioning of the $\mathrm{Ca}^{2+}$ clock is especially important for studies of the arrhythmia. Extraordinary fluctuations of the internal $\mathrm{Ca}^{2+}$-clock can disturb of self-oscillatory activity of the pacemaker cells, which can be an arrhythmogenic factor for the entire myocardium. In Fig. 3 timeseries of $C a_{S S}$ for different values of $k$ are presented. In case of the absence of coupling be- tween RyRs a high variance of $\mathrm{Ca}^{2+}$ sparks is observed. Switching on coupling $(k>0)$ leads to the increase of the sparks amplitude and to the decrease of $C a_{S S a m p}$ range (Fig.4). The deviation from the mean sparks amplitude decreased with the increase of $k$ value. It means that RyRs coupling leads to the stability of spontaneous sparks in the amplitude and in the frequency.

Further increase of the parameter $k$ value $(k>1.2)$ caused a sudden stop of $\mathrm{Ca}^{2+}$-clock oscillations. It is manifested in the appearance of a steady cluster of opened RyRs.

\section{Discussion}

We have demonstrated that the simple biophysically reasonable Electron-Conformational model is a novel approach for RyRs stochastic dynamics description as well as allosteric/conformational coupling between RyRs. Integrated to the $\mathrm{Ca}^{2+}$ dynamics model, this theory also can describe $\mathrm{Ca}^{2+}$-mediated RyRs coupling.

For sure, our model requires further developments like taking into account a complex structure of the $\mathrm{Ca}^{2+}$ release system as well as RyRs non-uniform spatial arrangement. However, on this stage we are able to describe $\mathrm{Ca}^{2+}$ sparks initiation-spread-termination process in a single $\mathrm{RU}$ and to determine the conditions for the periodic $\mathrm{Ca}^{2+}$ release disturbances.

We show that RyRs cooperativity is an important factor, which should be taken into account in $\mathrm{Ca}^{2+}$ sparks simulations in cardiac cells. RyRs coupling leads to sparks stability in the amplitude and frequency.

Also we observed a novel effect of the sudden stop of the periodic $\mathrm{Ca}^{2+}$ releases which can lead to $\mathrm{Ca}^{2+}$ leak and further cell functioning disturbances. both strong enough $\mathrm{Ca}^{2+}$-mediated coupling and conformational coupling between RyRs can be a reason of $\mathrm{Ca}^{2+}$ leak from the SR. Special genetic mutations of RyRs can be a reason of diverse diseases (e.g. catecholaminergic polymorphic ventricular tachycardia (CPVT)) [5].

\section{Acknowledgements}

The project is supported by RFBR grant 16-34-60223. The work was carried out within the framework of the IIF UrB RAS theme No AAAA-A18-118020590031-8 and RF Government Act 211 of March 16, 2013 (agreement 02.A03.21.0006).

\section{References}

[1] Cheng H, Lederer W, Cannell M. Calcium sparks: elementary events underlying excitation-contraction coupling in heart muscle. Science 1993;262(5134):740-744. 
[2] Wehrens X, Lehnart S, Marks A. Intracellular calcium release and cardiac disease. Annual Review Physiology 2005; 67:69-98.

[3] Marx S, Gaburjakova J, Gaburjakova M, et al. Coupled gating between cardiac calcium release channels (ryanodine receptors). Circulation research 2001;88(11):1151-1158.

[4] Asghari P, Scriven D, Sanatani S, et al. Non-uniform and variable arrangements of ryanodine receptors within mammalian ventricular couplons. Circulation research 2014;252262.

[5] Williams G, Chikando A, Tuan HT, et al. Dynamics of calcium sparks and calcium leak in the heart. Biophysical journal 2011;101(6):1287-1296.

[6] Lakatta E, Maltsev V, Vinogradova T. A coupled system of intracellular $\mathrm{Ca}^{2+}$ clocks and surface membrane voltage clocks controls the timekeeping mechanism of the heart's pacemaker. Circulation research 2010;106(4):659-673.

[7] Moskvin A, Ryvkin A, Solovyova O, et al. Electronconformational transformations in nanoscopic RyR channels governing both the heart's contraction and beating. JETP letters 2011;93(7):403-408.

[8] Moskvin A, Philipiev M, Solovyova O, et al. Electronconformational model of ryanodine receptor lattice dynamics. Progress in biophysics and molecular biology 2006; 90(1-3):88-103.

[9] Ryvkin A, Zorin N, Moskvin A, et al. The interaction of the membrane and calcium oscillators in cardiac pacemaker cells: Mathematical modeling. Biophysics 2015;60(6):946952.

Address for correspondence:

Name

Ryvkin Alexander 620000, Instiute of Immunology Physiology UrB RAS, Pervomayskaya 106, Ekarerinburg, Russia E-mail alex-ryvkin@ya.ru 